\title{
UNUSUAL LIMIT THEOREMS FOR THE DIFFERENCE OF ORDER STATISTICS FROM A PARETO
}

\author{
ANDRÉ ADLER
}

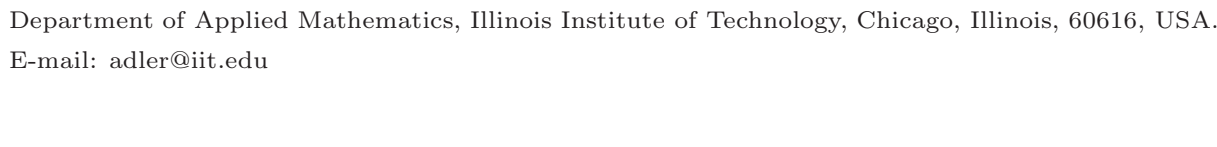

\begin{abstract}
In this paper we establish various limit theorems for the difference in order statistics from a sample from the Pareto distribution. The underlying density is $f(x)=x^{-2} I(x \geq 1)$. We look at both fixed and slowly increasing samples sizes. For our strong and weak laws of large numbers the first moment will be infinite and for our central limit theorem the second moment will be infinite. These theorems are quite unusual since the usual moment conditions do not hold. In order to achieve these results we must attach weights to these random variables and find these appropriate weights and norming sequences in order to establish our results.
\end{abstract}

\section{Introduction}

We establish various limit theorems for weighted sums of the difference of order statistics from a sample of $m_{n}$ random variables from a Pareto distribution. In some cases $m_{n}$ is fixed in others it will grow slowly towards infinity. In the previous paper [4] we looked at the largest minus the smallest order statistics from this very distribution. Now we look at any difference. And we also examine a Central Limit Theorem when we select the second biggest order statistic. These unusual results occur when we select either of the two larger order statistics.

These theorems are valuable. The underlying distribution does not possess a first and hence a second moment. The variance is infinite. So how

Received March 21, 2021.

AMS Subject Classification: 60F05, 60F15.

Key words and phrases: Almost sure convergence, order statistics, strong laws of large numbers, exact strong laws, weak law of large numbers, central limit theorems. 
does one measure the spread of these types of random variables. The conventional wisdom is to look at the range and divide it by four. The range is the largest order statistic minus the smallest order statistic. That's why we need to examine the differences of order statistics. But what happens if we need to throw away outliers? In that setting we may want the difference between other sets of order statistics and that is precisely what we are doing in this paper. And we only look at the difficult cases. We need not discuss the differences of order statistics when the difference has a finite moment.

We may also want to know how long certain equipment will last. If we have a dozen batteries and the system does fail only after the fifth one dies, but does grow weaker after the third one is dead, then we want to measure the difference between the third and fifth order statistics. Also, for married couples the time between the two deaths does matter. An insurance policy will only start after the second death. So, the difference matters once again.

The fascination with order statistics from the Pareto distribution and unusual limit theorems can be traced back to [2]. Next came the examination of the ratios of order statistics which can be found in [3]. There have been many extensions by many people, just two recent ones are [9] and [12].

The interest in finding a way to balance sums of random variables that do not possess a finite expectation with a sequence of constants dates back to the St. Petersburg game. The partial sum can be considered the winnings from a game at some point in time, while the sequence of constants would be the entrance fee at that same point in time. This phenomenon has gone by the name "fair games" problem and more recently "exact strong laws". Which is more appropriate when one wants almost sure convergence. Feller, [7], established a weak law for the St. Peterburg game, see page 252. But that weak law does not allow almost sure convergence and just like Theorem 6 , it allows the the gambler an unfair advantage. The almost sure upper limit in Feller's theorem is infinity and the almost sure lower limit is one. That is certainly not fair for the house. This is why we need to study Exact Strong Laws, one recent paper on such limit theorems is [8]. Others also interested in the infinite mean case are [10], [11] and [13]. But there are many more people who are now examining this odd behaviour.

We need to say that the constant $C$ used in the proofs denotes a generic real number that is not necessarily the same in each appearance. It is usually used as an upper bound in order to establish the convergence of our various 
series. And it also can be used as a generic lower bound for a divergence series. Also, we define $\lg x=\ln (\max \{e, x\})$ and $\lg _{2} x=\lg (\lg x)$, which is not a logarithm with a base of 2 . Likewise $\lg _{3} x=\lg (\lg (\lg x))$.

\section{Preliminary Results}

The underlying distribution is the classic Pareto, $f(x)=x^{-2} I(x \geq 1)$. We then take $n$ samples of size $m$ from this distribution. We start with $\left\{X_{i j}, 1 \leq i \leq n, 1 \leq j \leq m\right\}$ as independent and identically Pareto distributed random variables. The order statistics are no longer independent and they are denoted by $\left\{X_{i(1)}, \ldots, X_{i(m)}\right\}$, where $X_{i(1)} \leq X_{i(2)} \leq \cdots \leq$ $X_{i(m)}$. Next, we observe two order statistics from each sample, $X_{i(s)}$ and $X_{i(t)}$, where $1 \leq s<t \leq m$ and $i=1,2, \ldots n$. The two interesting cases are when $t=m$ or $t=m-1$.

From these two random variables we obtain the difference, $D_{i}=X_{i(t)}-$ $X_{i(s)}$. Let

$$
C_{s t m}=\frac{m !}{(s-1) !(t-s-1) !(m-t) !}
$$

which is the constant term in the joint density of two order statistics. In order to get the density of $D_{i}$, we first obtain the joint density of $X_{i(s)}$ and $X_{i(t)}$, which is

$$
\begin{aligned}
& f\left(x_{s}, x_{t}\right) \\
& =C_{s t m}\left[F\left(x_{s}\right)\right]^{s-1} f\left(x_{s}\right)\left[F\left(x_{t}\right)-F\left(x_{s}\right)\right]^{t-s-1} f\left(x_{t}\right)\left[1-F\left(x_{t}\right)\right]^{m-t} \\
& =C_{s t m}\left[1-\frac{1}{x_{s}}\right]^{s-1} \frac{1}{x_{s}^{2}}\left[\left(1-\frac{1}{x_{t}}\right)-\left(1-\frac{1}{x_{s}}\right)\right]^{t-s-1} \frac{1}{x_{t}^{2}}\left[\frac{1}{x_{t}}\right]^{m-t} I\left(1 \leq x_{s} \leq x_{t}\right) \\
& =C_{s t m}\left[1-\frac{1}{x_{s}}\right]^{s-1} \frac{1}{x_{s}^{2}}\left[\frac{1}{x_{s}}-\frac{1}{x_{t}}\right]^{t-s-1}\left[\frac{1}{x_{t}}\right]^{m-t+2} I\left(1 \leq x_{s} \leq x_{t}\right) .
\end{aligned}
$$

Next, let $w=x_{s}$ and $d=x_{t}-x_{s}$. The Jacobian is one and the joint density of $W$ and $D_{i}$ is

$$
f(w, d)=C_{s t m}\left(1-\frac{1}{w}\right)^{s-1} \frac{d^{t-s-1}}{w^{t-s+1}(w+d)^{m-s+1}} I(w \geq 1) I(d \geq 0) .
$$


Integrating out the dummy variable, $w$, we see that the density of $D_{i}$ is

$$
\begin{aligned}
f_{D_{i}}(d) & =C_{s t m} d^{t-s-1} \int_{1}^{\infty}\left(1-\frac{1}{w}\right)^{s-1} \frac{d w}{w^{t-s+1}(w+d)^{m-s+1}} \\
& =C_{s t m} \frac{d^{t-s-1}}{d^{m-s+1}} \int_{1}^{\infty}\left(1-\frac{1}{w}\right)^{s-1} \frac{d^{m-s+1} d w}{w^{t-s+1}(w+d)^{m-s+1}} \\
& \sim C_{s t m} d^{t-m-2} \int_{1}^{\infty}\left(1-\frac{1}{w}\right)^{s-1} \frac{d w}{w^{t-s+1}}
\end{aligned}
$$

where $d \geq 0$. Next, let $x=1 / w$ and use the Beta distribution to find the limiting distribution of our difference. Using that substitution we have

$$
\begin{aligned}
\int_{1}^{\infty}\left(1-\frac{1}{w}\right)^{s-1} \frac{d w}{w^{t-s+1}} & =\int_{1}^{0}(1-x)^{s-1} x^{t-s+1}\left(-x^{-2}\right) d x \\
& =\int_{0}^{1}(1-x)^{s-1} x^{t-s-1} d x=\frac{\Gamma(s) \Gamma(t-s)}{\Gamma(t)} .
\end{aligned}
$$

Thus

$$
\begin{aligned}
f_{D_{i}}(d) & \sim \frac{\Gamma(s) \Gamma(t-s)}{\Gamma(t)} \cdot C_{s t m} \cdot d^{t-m-2} \\
& =\frac{\Gamma(s) \Gamma(t-s)}{\Gamma(t)} \cdot \frac{m !}{(s-1) !(t-s-1) !(m-t) !} \cdot d^{t-m-2} \\
& =\frac{(s-1) !(t-s-1) !}{(t-1) !} \cdot \frac{m !}{(s-1) !(t-s-1) !(m-t) !} \cdot d^{t-m-2} \\
& =\frac{m !}{(m-t) !(t-1) !} \cdot d^{t-m-2} .
\end{aligned}
$$

which is free of $s$, but naturally, not free of $t$ nor $m$. With all that accomplished, we can now obtain our strong laws, weak laws and central limit theorem. When $t=m$ we will have our unusual strong laws and when $t=m-1$ we will have our unusual central limit theorem. When $t \leq m-2$ all our classic limit theorems exist since the second moment of our differences exist. This paper addresses just the difficult cases.

\section{Strong Laws}

When the larger of our order statistics is the maximum, then the expectation of our random variables is infinite. This section establishes strong laws for 
these type of random variables. We start by observing a fixed sample size with $t=m$.

Theorem 1. Let $\left\{X_{i 1}, \ldots, X_{i m}\right\}$ be i.i.d. random variables from the Pareto distribution and set $D_{i}=X_{i(m)}-X_{i\left(s_{i}\right)}$. For any $1 \leq s_{i} \leq m-1$ and any $\alpha>0$ we have

$$
\lim _{n \rightarrow \infty} \frac{\sum_{i=1}^{n} \frac{(\lg i)^{\alpha-2}}{i} D_{i}}{(\lg n)^{\alpha}}=\frac{m}{\alpha} \quad \text { almost surely. }
$$

Proof. Since $f_{D}(x) \sim m x^{-2}$, it follows that $x P\{D>x\} \sim m$. By applying Example 2 from [1] the conclusion follows.

As in [4] we examine what happens as the sample size increases at a particular rate. Here we select our largest order statistic from each sample, $t_{i}=m_{i}$, for all $i \geq 1$. But the smaller order statistic can be chosen as any other one within that sample and not necessarily the same each time.

Theorem 2. Let $\left\{X_{i 1}, \ldots, X_{i m_{i}}\right\}$ be i.i.d. random variables from the Pareto distribution and set $D_{i}=X_{i\left(m_{i}\right)}-X_{i\left(s_{i}\right)}$. For any $1 \leq s_{i} \leq m_{i}-1$ where $m_{n} \sim \gamma(\lg n)^{\beta}$, then for $\gamma, \beta$ and $\alpha+\beta+2$ all positive

$$
\lim _{n \rightarrow \infty} \frac{\sum_{i=1}^{n} \frac{(\lg i)^{\alpha}}{i} D_{i}}{(\lg n)^{\alpha+\beta+2}}=\frac{\gamma}{\alpha+\beta+2} \quad \text { almost surely. }
$$

Proof. Let $a_{n}=(\lg n)^{\alpha} / n, b_{n}=(\lg n)^{\alpha+\beta+2}$ and $c_{n}=b_{n} / a_{n}=n(\lg n)^{\beta+2}$. We use the partition

$$
\begin{aligned}
\frac{1}{b_{n}} \sum_{i=1}^{n} a_{i} D_{i}= & \frac{1}{b_{n}} \sum_{i=1}^{n} a_{i}\left[D_{i} I\left(\left|D_{i}\right| \leq c_{i}\right)-E D_{i} I\left(\left|D_{i}\right| \leq c_{i}\right)\right] \\
& +\frac{1}{b_{n}} \sum_{i=1}^{n} a_{i} D_{i} I\left(\left|D_{i}\right|>c_{i}\right) \\
& +\frac{1}{b_{n}} \sum_{i=1}^{n} a_{i} E D_{i} I\left(\left|D_{i}\right| \leq c_{i}\right) .
\end{aligned}
$$

The first term vanishes almost surely by the Khintchine-Kolmogorov 
Convergence Theorem, see page 113 of [6], and Kronecker's lemma since

$$
\begin{aligned}
\sum_{n=1}^{\infty} \frac{1}{c_{n}^{2}} E D_{n}^{2} I\left(\left|D_{n}\right| \leq c_{n}\right) & <C \sum_{n=1}^{\infty} \frac{1}{c_{n}^{2}} \int_{1}^{c_{n}} m_{n} d x \\
& <C \sum_{n=1}^{\infty} \frac{m_{n}}{c_{n}}<C \sum_{n=1}^{\infty} \frac{1}{n(\lg n)^{2}}<\infty .
\end{aligned}
$$

The second term vanishes, with probability one, by the Borel-Cantelli lemma since

$$
\sum_{n=1}^{\infty} P\left\{\left|D_{n}\right|>c_{n}\right\}<C \sum_{n=1}^{\infty} \int_{c_{n}}^{\infty} \frac{m_{n} d x}{x^{2}}=C \sum_{n=1}^{\infty} \frac{m_{n}}{c_{n}}<\infty .
$$

Thus, our almost sure limit follows from the last term in our partition

$$
\begin{aligned}
\frac{\sum_{i=1}^{n} a_{i} E D_{i} I\left(\left|D_{i}\right| \leq c_{i}\right)}{b_{n}} & \sim \frac{\sum_{i=1}^{n} \frac{(\lg i)^{\alpha}}{i} \int_{1}^{c_{i}} \frac{m_{i} d x}{x}}{(\lg n)^{\alpha+\beta+2}} \\
& =\frac{\sum_{i=1}^{n} \frac{(\lg i)^{\alpha}}{i} m_{i} \lg c_{i}}{(\lg n)^{\alpha+\beta+2}} \\
& \sim \frac{\gamma \sum_{i=1}^{n} \frac{(\lg i)^{\alpha+\beta+1}}{i}}{(\lg n)^{\alpha+\beta+2}} \\
& \rightarrow \frac{\gamma}{\alpha+\beta+2}
\end{aligned}
$$

which concludes this proof.

We continue to shrink, both our weights and our norming sequence to show there are many exact strong laws in this setting.

Theorem 3. Let $\left\{X_{i 1}, \ldots, X_{i m_{i}}\right\}$ be i.i.d. random variables from the Pareto distribution and set $D_{i}=X_{i\left(m_{i}\right)}-X_{i\left(s_{i}\right)}$. For any $1 \leq s_{i} \leq m_{i}-1$ where $m_{n} \sim \gamma(\lg n)^{\beta}$, then for $\gamma$ and $\beta$ both positive

$$
\lim _{n \rightarrow \infty} \frac{\sum_{i=1}^{n} \frac{1}{i(\lg i)^{\beta+2}} D_{i}}{\lg _{2} n}=\gamma \quad \text { almost surely. }
$$

Proof. Let $a_{n}=1 /\left(n(\lg n)^{\beta+2}\right), b_{n}=\lg _{2} n$ and $c_{n}=b_{n} / a_{n}=n(\lg n)^{\beta+2} \lg _{2} n$. 
Again, we use the partition

$$
\begin{aligned}
\frac{1}{b_{n}} \sum_{i=1}^{n} a_{i} D_{i}= & \frac{1}{b_{n}} \sum_{i=1}^{n} a_{i}\left[D_{i} I\left(\left|D_{i}\right| \leq c_{i}\right)-E D_{i} I\left(\left|D_{i}\right| \leq c_{i}\right)\right] \\
& +\frac{1}{b_{n}} \sum_{i=1}^{n} a_{i} D_{i} I\left(\left|D_{i}\right|>c_{i}\right) \\
& +\frac{1}{b_{n}} \sum_{i=1}^{n} a_{i} E D_{i} I\left(\left|D_{i}\right| \leq c_{i}\right) .
\end{aligned}
$$

The first term vanishes almost surely by the Khintchine-Kolmogorov Convergence Theorem and Kronecker's lemma since

$$
\begin{aligned}
\sum_{n=1}^{\infty} \frac{1}{c_{n}^{2}} E D_{n}^{2} I\left(\left|D_{n}\right| \leq c_{n}\right) & <C \sum_{n=1}^{\infty} \frac{1}{c_{n}^{2}} \int_{1}^{c_{n}} m_{n} d x \\
& <C \sum_{n=1}^{\infty} \frac{m_{n}}{c_{n}}<C \sum_{n=1}^{\infty} \frac{1}{n(\lg n)^{2} \lg _{2} n}<\infty .
\end{aligned}
$$

The second term vanishes, with probability one, by the Borel-Cantelli lemma since

$$
\sum_{n=1}^{\infty} P\left\{\left|D_{n}\right|>c_{n}\right\}<C \sum_{n=1}^{\infty} \int_{c_{n}}^{\infty} \frac{m_{n} d x}{x^{2}}=C \sum_{n=1}^{\infty} \frac{m_{n}}{c_{n}}<\infty .
$$

Thus, our almost sure limit follows from the last term in our partition

$$
\begin{aligned}
\frac{\sum_{i=1}^{n} a_{i} E D_{i} I\left(\left|D_{i}\right| \leq c_{i}\right)}{b_{n}} & \sim \frac{\sum_{i=1}^{n} \frac{1}{i(\lg i)^{\beta+2}} \int_{1}^{c_{i}} \frac{m_{i} d x}{x}}{\lg _{2} n} \\
& =\frac{\sum_{i=1}^{n} \frac{1}{i(\lg i)^{\beta+2}} m_{i} \lg c_{i}}{\lg _{2} n} \\
& \sim \frac{\gamma \sum_{i=1}^{n} \frac{1}{i(\lg i)^{\beta+2}}(\lg i)^{\beta} \lg i}{\lg _{2} n} \\
& =\frac{\gamma \sum_{i=1}^{n} \frac{1}{i \lg i}}{\lg _{2} n} \\
& \rightarrow \gamma
\end{aligned}
$$

which concludes this proof. 
We can continue to get smaller and smaller weights and norming sequences. We conclude with one such result.

Theorem 4. Let $\left\{X_{i 1}, \ldots, X_{i m_{i}}\right\}$ be i.i.d. random variables from the Pareto distribution and set $D_{i}=X_{i\left(m_{i}\right)}-X_{i\left(s_{i}\right)}$. For any $1 \leq s_{i} \leq m_{i}-1$ where $m_{n} \sim \gamma(\lg n)^{\beta}$, then for $\gamma$ and $\beta$ both positive

$$
\lim _{n \rightarrow \infty} \frac{\sum_{i=1}^{n} \frac{1}{i(\lg i)^{\beta+2} \lg _{2} i} D_{i}}{\lg _{3} n}=\gamma \quad \text { almost surely. }
$$

Proof. Let $a_{n}=1 /\left(n(\lg n)^{\beta+2} \lg _{2} n\right), b_{n}=\lg _{3} n$ and $c_{n}=b_{n} / a_{n}=$ $n(\lg n)^{\beta+2} \lg _{2} n \lg _{3} n$. Once again, we use the partition

$$
\begin{aligned}
\frac{1}{b_{n}} \sum_{i=1}^{n} a_{i} D_{i}= & \frac{1}{b_{n}} \sum_{i=1}^{n} a_{i}\left[D_{i} I\left(\left|D_{i}\right| \leq c_{i}\right)-E D_{i} I\left(\left|D_{i}\right| \leq c_{i}\right)\right] \\
& +\frac{1}{b_{n}} \sum_{i=1}^{n} a_{i} D_{i} I\left(\left|D_{i}\right|>c_{i}\right) \\
& +\frac{1}{b_{n}} \sum_{i=1}^{n} a_{i} E D_{i} I\left(\left|D_{i}\right| \leq c_{i}\right) .
\end{aligned}
$$

The first term vanishes almost surely by the Khintchine-Kolmogorov Convergence Theorem and Kronecker's lemma since

$$
\begin{aligned}
\sum_{n=1}^{\infty} \frac{1}{c_{n}^{2}} E D_{n}^{2} I\left(\left|D_{n}\right| \leq c_{n}\right) & <C \sum_{n=1}^{\infty} \frac{1}{c_{n}^{2}} \int_{1}^{c_{n}} m_{n} d x \\
& <C \sum_{n=1}^{\infty} \frac{m_{n}}{c_{n}}<C \sum_{n=1}^{\infty} \frac{1}{n(\lg n)^{2} \lg _{2} n \lg _{3} n}<\infty
\end{aligned}
$$

The second term vanishes, with probability one, by the Borel-Cantelli lemma since

$$
\sum_{n=1}^{\infty} P\left\{\left|D_{n}\right|>c_{n}\right\}<C \sum_{n=1}^{\infty} \int_{c_{n}}^{\infty} \frac{m_{n} d x}{x^{2}}=C \sum_{n=1}^{\infty} \frac{m_{n}}{c_{n}}<\infty .
$$


Thus, our almost sure limit follows from the last term in our partition

$$
\begin{aligned}
\frac{\sum_{i=1}^{n} a_{i} E D_{i} I\left(\left|D_{i}\right| \leq c_{i}\right)}{b_{n}} & \sim \frac{\sum_{i=1}^{n} \frac{1}{i(\lg i)^{\beta+2} \lg _{2} i} \int_{1}^{c_{i}} \frac{m_{i} d x}{x}}{\lg _{3} n} \\
& =\frac{\sum_{i=1}^{n} \frac{1}{i(\lg i)^{\beta+2} \lg _{2} i} m_{i} \lg c_{i}}{\lg _{3} n} \\
& \sim \frac{\gamma \sum_{i=1}^{n} \frac{1}{i(\lg i)^{\beta+2} \lg _{2} i}(\lg i)^{\beta} \lg i}{\lg _{3} n} \\
& =\frac{\gamma \sum_{i=1}^{n} \frac{1}{i \lg ^{2} \lg _{2} i}}{\lg _{3} n} \\
& \rightarrow \gamma
\end{aligned}
$$

which concludes this proof.

\section{Weak Laws and One Sided Strong Laws}

We saw in the last section that it was sufficient for the weights to be of the form $a_{n}=1 / n$. And we can increase them by a slowly varying function, such as the logarithm, but no more than that. In this section we show that it is necessary for our weights to be of this form in order to have an Exact Strong Law.

Theorem 5. Let $\left\{X_{i 1}, \ldots, X_{i m_{i}}\right\}$ be i.i.d. random variables from the Pareto distribution and set $D_{i}=X_{i\left(m_{i}\right)}-X_{i\left(s_{i}\right)}$, where $1 \leq s_{i} \leq m_{i}-1$, with $m_{n} \sim \gamma(\lg n)^{\beta}$. If $\alpha>-1, \gamma>0$ and $\beta>0$, then for any slowly varying function $L(x)$

$$
\frac{\sum_{i=1}^{n} L(i) i^{\alpha} D_{i}}{L(n)(\lg n)^{\beta+1} n^{\alpha+1}} \stackrel{P}{\rightarrow} \frac{\gamma}{\alpha+1}
$$

Proof. Let $a_{i}=L(i) i^{\alpha}$ and $b_{n}=L(n)(\lg n)^{\beta+1} n^{\alpha+1}$. We will use the Weak Law from page 356 of [6]. Let $\epsilon>0$

$$
\begin{aligned}
\sum_{i=1}^{n} P\left\{a_{i} D_{i} / b_{n}>\epsilon\right\} & <C \sum_{i=1}^{n} \int_{\epsilon b_{n} / a_{i}}^{\infty} \frac{m_{i} d x}{x^{2}}<C \sum_{i=1}^{n} \frac{m_{i} a_{i}}{b_{n}} \\
& <\frac{C \sum_{i=1}^{n}(\lg i)^{\beta} L(i) i^{\alpha}}{L(n)(\lg n)^{\beta+1} n^{\alpha+1}}<\frac{C L(n)(\lg n)^{\beta} n^{\alpha+1}}{L(n)(\lg n)^{\beta+1} n^{\alpha+1}}=\frac{C}{\lg n} \rightarrow 0 .
\end{aligned}
$$


As for the variance term, we have

$\sum_{i=1}^{n} V\left[\frac{a_{i} D_{i}}{b_{n}} I\left(\left|\frac{a_{i} D_{i}}{b_{n}}\right|<1\right)\right]<C \sum_{i=1}^{n}\left(\frac{a_{i}^{2}}{b_{n}^{2}}\right) \int_{0}^{b_{n} / a_{i}} m_{i} d x<C \sum_{i=1}^{n} \frac{m_{i} a_{i}}{b_{n}} \rightarrow 0$

once again.

Next, we must compute the expectation from that theorem

$$
\begin{aligned}
\sum_{i=1}^{n} E & {\left[\frac{a_{i} D_{i}}{b_{n}} I\left(\left|\frac{a_{i} D_{i}}{b_{n}}\right|<1\right)\right] } \\
= & b_{n}^{-1} \sum_{i=1}^{n} m_{i} a_{i} \lg \left(b_{n}^{-1} \sum_{i=1}^{n} a_{i}\right)=b_{n}^{-1} \int_{i=1}^{n} m_{i} a_{i} \lg \left(b_{n}\right)-b_{n}^{-1} \sum_{i=1}^{n} m_{i} a_{i} \lg \left(a_{i}\right) .
\end{aligned}
$$

It's interesting that both of these terms are equally important

$$
\begin{aligned}
b_{n}^{-1} \sum_{i=1}^{n} m_{i} a_{i} \lg \left(b_{n}\right) & \sim \frac{\gamma \sum_{i=1}^{n}(\lg i)^{\beta} L(i) i^{\alpha}\left[\lg (L(n))+(\beta+1) \lg _{2} n+(\alpha+1) \lg n\right]}{L(n)(\lg n)^{\beta+1} n^{\alpha+1}} \\
& \sim \frac{\gamma(\alpha+1) \sum_{i=1}^{n}(\lg i)^{\beta} L(i) i^{\alpha} \lg n}{L(n)(\lg n)^{\beta+1} n^{\alpha+1}} \\
& \sim \frac{\gamma(\alpha+1)\left[(\lg n)^{\beta} L(n)\left(\frac{n^{\alpha+1}}{\alpha+1}\right)\right] \lg n}{L(n)(\lg n)^{\beta+1} n^{\alpha+1}}=\gamma
\end{aligned}
$$

and the other term is

$$
\begin{aligned}
b_{n}^{-1} \sum_{i=1}^{n} m_{i} a_{i} \lg \left(a_{i}\right) & \sim \frac{\gamma \sum_{i=1}^{n}(\lg i)^{\beta} L(i) i^{\alpha}[\lg (L(i))+\alpha \lg i]}{L(n)(\lg n)^{\beta+1} n^{\alpha+1}} \\
& \sim \frac{\alpha \gamma \sum_{i=1}^{n}(\lg i)^{\beta+1} L(i) i^{\alpha}}{L(n)(\lg n)^{\beta+1} n^{\alpha+1}} \\
& \sim \frac{\alpha \gamma\left[(\lg n)^{\beta+1} L(n)\left(\frac{n^{\alpha+1}}{\alpha+1}\right)\right]}{L(n)(\lg n)^{\beta+1} n^{\alpha+1}} \\
& =\frac{\alpha \gamma}{\alpha+1} .
\end{aligned}
$$

Combining these two terms, we see that our limit is

$$
\gamma-\frac{\alpha \gamma}{\alpha+1}=\frac{\gamma}{\alpha+1}
$$

which concludes this proof. 
In this setting we cannot get an Exact Strong Law. The almost sure lower limit agrees with our Weak Law, but the almost sure upper limit is infinity. This is precisely what happens with the famous St Petersburg game.

Theorem 6. Let $\left\{X_{i 1}, \ldots, X_{i m_{i}}\right\}$ be i.i.d. random variables from the Pareto distribution and set $D_{i}=X_{i\left(m_{i}\right)}-X_{i\left(s_{i}\right)}$, where $1 \leq s_{i} \leq m_{i}-1$, with $m_{n} \sim \gamma(\lg n)^{\beta}$. If $\alpha>-1, \gamma>0$ and $\beta>0$, then for any slowly varying function $L(x)$

$$
\liminf _{n \rightarrow \infty} \frac{\sum_{i=1}^{n} L(i) i^{\alpha} D_{i}}{L(n)(\lg n)^{\beta+1} n^{\alpha+1}}=\frac{\gamma}{\alpha+1} \quad \text { almost surely }
$$

and

$$
\limsup _{n \rightarrow \infty} \frac{\sum_{i=1}^{n} L(i) i^{\alpha} D_{i}}{L(n)(\lg n)^{\beta+1} n^{\alpha+1}}=\infty \quad \text { almost surely. }
$$

Proof. In this proof we set $a_{n}=L(n) n^{\alpha}, b_{n}=L(n)(\lg n)^{\beta+1} n^{\alpha+1}, c_{n}=$ $b_{n} / a_{n}=n(\lg n)^{\beta+1}$ and we now introduce a new sequence $h_{n}=c_{n} /\left(\lg _{2} n\right)^{2}=$ $n(\lg n)^{\beta+1} /\left(\lg _{2} n\right)^{2}$.

Since we have convergence in probability from Theorem 5 , we can claim that

$$
\liminf _{n \rightarrow \infty} \frac{\sum_{i=1}^{n} L(i) i^{\alpha} D_{i}}{L(n)(\lg n)^{\beta+1} n^{\alpha+1}} \leq \frac{\gamma}{\alpha+1} \quad \text { almost surely. }
$$

Hence we need to prove that

$$
\liminf _{n \rightarrow \infty} \frac{\sum_{i=1}^{n} L(i) i^{\alpha} D_{i}}{L(n)(\lg n)^{\beta+1} n^{\alpha+1}} \geq \frac{\gamma}{\alpha+1} \quad \text { almost surely. }
$$

This is where the sequence $h_{n}$ comes into play. Clearly

$$
\begin{aligned}
b_{n}^{-1} \sum_{i=1}^{n} a_{i} D_{i} \geq & b_{n}^{-1} \sum_{i=1}^{n} a_{i} D_{i} I\left(0 \leq D_{i} \leq h_{i}\right) \\
= & b_{n}^{-1} \sum_{i=1}^{n} a_{i}\left[D_{i} I\left(0 \leq D_{i} \leq h_{i}\right)-E\left(D_{i} I\left(0 \leq D_{i} \leq h_{i}\right)\right)\right] \\
& +b_{n}^{-1} \sum_{i=1}^{n} a_{i} E\left(D_{i} I\left(0 \leq D_{i} \leq h_{i}\right)\right) .
\end{aligned}
$$

The first term vanishes almost surely by the Khintchine-Kolmogorov Con- 
vergence Theorem and Kronecker's lemma since

$$
\begin{aligned}
\sum_{n=1}^{\infty} \frac{1}{c_{n}^{2}} E D_{n}^{2} I\left(0 \leq D_{n} \leq h_{n}\right) & <C \sum_{n=1}^{\infty} \frac{1}{c_{n}^{2}} \int_{0}^{h_{n}} m_{n} d x \\
& =C \sum_{n=1}^{\infty} \frac{m_{n} h_{n}}{c_{n}^{2}}<C \sum_{n=1}^{\infty} \frac{(\lg n)^{\beta} n(\lg n)^{\beta+1} /\left(\lg _{2} n\right)^{2}}{\left(n(\lg n)^{\beta+1}\right)^{2}} \\
& =C \sum_{n=1}^{\infty} \frac{1}{n \lg n\left(\lg _{2} n\right)^{2}}<\infty
\end{aligned}
$$

And the limit of the second term is

$$
\begin{aligned}
b_{n}^{-1} \sum_{i=1}^{n} a_{i} E\left(D_{i} I\left(0 \leq D_{i} \leq h_{i}\right)\right) & \sim b_{n}^{-1} \sum_{i=1}^{n} a_{i} \int_{1}^{h_{i}} \frac{m_{i} d x}{x}=b_{n}^{-1} \sum_{i=1}^{n} a_{i} m_{i} \lg \left(h_{i}\right) \\
& \sim \frac{\sum_{i=1}^{n} L(i) i^{\alpha} \gamma(\lg i)^{\beta}\left[\lg i+(\beta+1) \lg _{2} i-2 \lg _{3} i\right]}{L(n)(\lg n)^{\beta+1} n^{\alpha+1}} \\
& \sim \frac{\gamma \sum_{i=1}^{n} L(i) i^{\alpha}(\lg i)^{\beta+1}}{L(n)(\lg n)^{\beta+1} n^{\alpha+1}} \\
& \sim \frac{\gamma L(n)\left(\frac{n^{\alpha+1}}{\alpha+1}\right)(\lg n)^{\beta+1}}{L(n)(\lg n)^{\beta+1} n^{\alpha+1}} \\
& =\frac{\gamma}{\alpha+1} .
\end{aligned}
$$

Thus showing that the almost sure lower limit is indeed $\gamma /(\alpha+1)$.

The upper limit is easier. Here, we use $C$ in the opposite direction, since we want this series to diverge. Let $M$ be any positive real number, then

$$
\begin{aligned}
\sum_{n=1}^{\infty} P\left\{\frac{a_{n} D_{n}}{b_{n}}>M\right\} & >C \sum_{n=1}^{\infty} \int_{M c_{n}}^{\infty} \frac{m_{n} d x}{x^{2}} \\
& >C \sum_{n=1}^{\infty} \frac{m_{n}}{c_{n}} \\
& >C \sum_{n=1}^{\infty} \frac{(\lg n)^{\beta}}{n(\lg n)^{\beta+1}} \\
& =C \sum_{n=1}^{\infty} \frac{1}{n \lg n}=\infty .
\end{aligned}
$$


Thus

$$
\limsup _{n \rightarrow \infty} \frac{\sum_{i=1}^{n} a_{i} D_{i}}{b_{n}} \geq \limsup _{n \rightarrow \infty} \frac{a_{n} D_{n}}{b_{n}}=\infty \text { almost surely }
$$

which concludes this proof.

\section{Central Limit Theorem}

Finally we look at the case where $E\left(D_{i}\right)<\infty$, but $E\left(D_{i}^{2}\right)=\infty$. This happens whenever we look at the second largest order statistic. Hence we conclude with a Central Limit Theorem for a fixed sample size, where $t=$ $m-1$, while once again the other order statistic can be anything smaller than $X_{i(m-1)}$. The only issue here, is in order to compute our centering in this Central Limit Theorem one must know which $X_{i\left(s_{i}\right)}$ we are choosing within each of our samples and also we would have to know the precise density of $D_{i}$, not just its tail behaviour. To make the sequence $\left\{D_{i}, i=1 \ldots n\right\}$ i.i.d. we would need to select the same smaller order statistic each time. We are only doing that to apply Theorem 4 from [5]. Without any doubt one can still obtain a Central Limit Theorem for nonidentically distributed differences of order statistics just as well.

There are three conditions that we need to meet in order to apply that theorem. One is trivial, it's that $x^{2} P\left\{D_{i}>x\right\}$ is slowly varying. The other two are

$$
G\left(\frac{B_{n}}{\min _{1 \leq i \leq n} a_{i}}\right) \sim G\left(\frac{B_{n}}{\max _{1 \leq i \leq n} a_{i}}\right)
$$

and for all $\epsilon>0$

$$
\sum_{i=1}^{n} P\left\{D_{i}>\epsilon B_{n} / a_{i}\right\}=o(1)
$$

where once again $a_{i}$ are our weights, but now $B_{n}$ is our norming sequence. The function $G(x)$ is either $E D_{i}^{2} I\left(D_{i} \leq x\right)$ or $\int_{0}^{x} 2 t P\left\{D_{i}>t\right\} d t$. In both cases one can see that since

$$
P\left\{D_{i}>x\right\} \sim \frac{m(m-1)}{2 x^{2}}
$$


we have

$$
G(x) \sim m(m-1) \lg x .
$$

The formula for $B_{n}$ is quite restrictive. It is $B_{n}^{2} \sim n G\left(B_{n}\right)$, which for us is $B_{n}^{2} \sim m(m-1) n \lg \left(B_{n}\right)$, which allows us to choose as our norming sequence

$$
B_{n}=\sqrt{\left(\begin{array}{c}
m \\
2
\end{array}\right) n \lg n .}
$$

For simplicity we will let $a_{i}=(\lg i)^{\alpha}$, which makes (10) trivial. But in order to satisfy (2) we will have to set $\alpha$ to be less than one-half.

Theorem 7. Let $\left\{X_{i 1}, \ldots, X_{i m}\right\}$ be i.i.d. random variables from the Pareto distribution and set $D_{i}=X_{i(m-1)}-X_{i(s)}$, where $1 \leq s \leq m-2$. If $\alpha<1 / 2$, then

$$
\frac{\sum_{i=1}^{n}(\lg i)^{\alpha}\left[D_{i}-\frac{m(m-s-1)}{m-s}\right]}{\sqrt{\left(\begin{array}{c}
m \\
2
\end{array}\right) n \lg n}} \stackrel{d}{\rightarrow} N(0,1) .
$$

Proof. Since $f_{D_{i}}(x) \sim m(m-1) x^{-3}$, it follows that $x^{2} P\{D>x\} \sim$ $m(m-1) / 2$. Thus $G(x) \sim m(m-1) \lg x$. That makes both sides of (11) asymptotically the same. Both $G\left(\frac{B_{n}}{\min _{1 \leq i \leq n} a_{i}}\right)$ and $G\left(\frac{B_{n}}{\max _{1 \leq i \leq n} a_{i}}\right)$ are approximately $(\lg n) / 2$. The more restrictive condition is (2), which is $\sum_{i=1}^{n}(\lg i)^{2 \alpha}=o(n \lg n)$, which holds whenever $\alpha<1 / 2$.

The centering sequence from [5] is

$$
A_{n}=\sum_{i=1}^{n}(\lg i)^{\alpha} E\left(D_{i}\right) .
$$

Since $D=X_{(m-1)}-X_{(s)}$, in order, pun intended, to obtain the expectation of $D$ it will be easier to obtain the expectation of our individual order statistics and then subtract. The density of the $k^{\text {th }}$ order statistic from our Pareto is

$$
f_{X_{(k)}}(x)=\frac{m !}{(k-1) !(m-k) !}\left(1-\frac{1}{x}\right)^{k-1}\left(\frac{1}{x}\right)^{m-k+2} I(x \geq 1) .
$$


Thus, by letting $u=1 / x$, we have

$$
\begin{aligned}
E\left(X_{(k)}\right) & =\frac{m !}{(k-1) !(m-k) !} \int_{1}^{\infty}\left(1-\frac{1}{x}\right)^{k-1}\left(\frac{1}{x}\right)^{m-k+1} d x \\
& =\frac{m !}{(k-1) !(m-k) !} \int_{0}^{1}(1-u)^{k-1} u^{m-k-1} d u \\
& =\frac{m !}{(k-1) !(m-k) !} \cdot \frac{\Gamma(k) \Gamma(m-k)}{\Gamma(m)} \\
& =\frac{m}{m-k} .
\end{aligned}
$$

This allows us to conclude that

$$
E(D)=E\left(X_{(m-1)}\right)-E\left(X_{(s)}\right)=m-\frac{m}{m-s}=\frac{m(m-s-1)}{m-s} .
$$

Then by applying Theorem 4 from [5] the conclusion follows.

And once again, it should be mentioned that whenever $t \leq m-2$, both the first two moments of our random variables exist. Thus the classic strong laws, weak laws and central limit theorems all hold.

\section{References}

1. A. Adler, Exact strong laws, Bull. Inst. Math. Acad. Sinica, 28 (2000), 141-166.

2. A. Adler, Exact strong laws for sums of order statistics from the Pareto distribution, Bull. Inst. Math. Acad. Sinica, 31 (2003), 181-193.

3. A. Adler, Exact strong laws for sums of ratios of order statistics from the Pareto distribution, Central European Journal of Mathematics, 4 (2006), No.1, 1-4. doi.org/10.1007/s11533-005-0001-6

4. A. Adler, Exact strong laws for the range, Bull. Inst. Math. Acad. Sinica New Series, 15 (2020), No.1, 53-61.

5. A. Adler and A. Rosalsky, Some Generalized Central Limit Theorems for Weighted Sums with Infinite Variance, Calcutta Statistical Association Bulletin, 38 (1989), 2742. doi.org/10.1177/0008068319890102

6. Y. S. Chow and H. Teicher, Probability Theory: Independence, Interchangeability, Martingales, third edition, Springer-Verlag, New York, 1997.

7. W. Feller, An Introduction to Probability Theory and Its Applications Volume 1, third edition, Wiley and Sons, New York, 1968. 
8. R. Giuliano and M. Hadjikyriakou, On Exact Laws of Large Numbers for Oppenheim Expansions with Infinite Mean, Journal of Theoretical Probability (2020). DOI: $10.1007 / \mathrm{s} 10959-020-01010-3$

9. P. Matula, A. Adler and P. Kurasinski, On exact strong laws of large numbers for ratios of random variables and their applications, Communications in Statistics- Theory and Methods, 49 (2020), No.13, 3153-3167.

10. T. Nakata, Exact laws of large numbers for independent Pareto random variables, Bull. Inst. Math. Acad. Sinica New Series, 13 (2017), No.4, 325-338.

11. H. Xu, X. Li, W. Yang and F. Xu, Laws of large numbers with infinite mean, Journal of mathematical inequalities, 13 (2019), No.2, 225-349.

12. S. F. Xu and Y. Miao, Some limit theorems for ratios of order statistics from uniform random variables, Journal of Mathematical Inequalities, (2017).

DOI 10.1186/s13660-017-1569-7

13. W. Yang, L. Yang, D. Wei, and S. Hu, The laws of large numbers for Pareto-type random variables with infinite means, Communications in Statistics- Theory and Methods, 48 (2019), No.12, 3044-3054. 